\title{
Immunomodulatory actions of methotrexate on T cells in juvenile idiopathic arthritis
}

\author{
Maja Bulatović Ćalasan*, Sebastiaan Vastert, Frederik Verweij, Nico Wulffraat, Femke van Wijk, Berent Prakken \\ From 7th European Workshop on Immune-Mediated Inflammatory Diseases \\ Noordwijk aan Zee, the Netherlands. 28-30 November 2012
}

\section{Introduction}

In the pathophysiology of juvenile idiopathic arthritis (JIA), T cells play an important role. The balance between immune activation, governed by effector $\mathrm{T}$ cells (Teff) and immune regulation, governed by regulatory $\mathrm{T}$ cells (Treg), is disturbed. Low-dose methotrexate (MTX) is the most commonly used drug to induce disease remission in JIA. Nevertheless, despite its use for decades as the prime antiinflammatory drug, its immunomodulatory actions in vivo remain poorly elucidated. We hypothesized that MTX restores the immune balance by increasing Treg number and suppressive function and decreasing Teff activation status and effector functions.

\section{Aim}

To determine the immunomodulatory effects of MTX on Treg and Teff in JIA during MTX treatment.

\section{Methods}

Peripheral blood mononuclear cells (PBMCs) of JIA patients were isolated before MTX start (T0), at 3 (T3) and 6 months (T6) during MTX treatment. Frequency and phenotype of CD4+FoxP3+ Treg were analyzed $e x$ vivo by flow cytometry, and their suppressive function was analyzed in CFSE suppression assays. Proliferation of CD4+ and CD8+ Teff was determined with CFSE, upon a 5 -day culture in the presence of a-CD3. Cytokine production was measured ex vivo upon short PMA/ionomycin stimulation by flow cytometry as well as in 5-day culture supernatants and in plasma by luminex.

\section{Results}

Treg frequency and suppressive capacity did not increase during MTX treatment. Frequency of naïve CD4+CD45RA + Teff increased, whereas the memory
CD45RO+ Teff decreased at T3 vs. T0. CD4+ and CD8+ Teff proliferation was significantly increased at T6 (mean: 75.3 +/- SD 14.9) vs. T0 (mean: 58.5 +/- SD 17.9) $(\mathrm{p}=0.002)$ in patients with good and poor MTX response. Ex vivo and supernatant cytokines showed no differences between time-points, however plasma IFN $\gamma$ was markedly increased at T6 vs. T3 and T0.

\section{Conclusion}

We observed no effects of MTX on Treg. In contrast to our hypothesis, Teff increased their proliferation during 6 months of MTX treatment, which was accompanied by increased plasma IFN $\gamma$. Taken together, this suggests immunomodulatory rather than immunosuppressive effects of low-dose MTX treatment in JIA. We are now investigating the role of antigen-presenting cells in increased $\mathrm{T}$ cell proliferation during MTX treatment.

Published: 28 November 2012

\section{doi:10.1186/1479-5876-10-S3-P40}

Cite this article as: Ćalasan et al:: Immunomodulatory actions of methotrexate on T cells in juvenile idiopathic arthritis. Journal of Translational Medicine 2012 10(Suppl 3):P40. 